\title{
Diagnosis, Prevalence, and Treatment of Halitosis
}

\author{
Andrea Zürcher • Marja L. Laine • Andreas Filippi
}

Published online: 30 September 2014

(C) Springer International Publishing AG 2014

\begin{abstract}
Halitosis affects a large part of the population. Many of those that are affected suffer from this problem for a long time before they visit a professional halitosis clinic. Further, halitosis creates social and psychological disadvantages. Although halitosis has multifactorial origins, in nine out of ten cases the source can be found within the oral cavity. The most frequent intra-oral causes are tongue coating, gingivitis, and periodontitis, or a combination of these. A general dental practitioner is often faced with patients suffering from halitosis in her/his dental practice and therefore should be able to manage it. Using standardized strategies, a high level of treatment success can be achieved. In the literature, several methods to determine halitosis and various possibilities for treatment are described. The present review summarizes diagnostics, prevalence, and current treatments of oral halitosis.
\end{abstract}

Keywords Halitosis - Bad breath · Psychogenic halitosis .

Diagnosis $\cdot$ Prevalence $\cdot$ Treatment

\section{Introduction}

Halitosis is a widespread problem in the general population. Due to increasing media exposure in recent years, halitosis has

A. Zürcher $(\bowtie) \cdot$ A. Filippi

Department of Oral Surgery, Oral Radiology and Oral Medicine, School of Dental Medicine, University of Basel, Hebelstrasse 3, 4056 Basel, Switzerland

e-mail: andrea.zuercher@unibas.ch

A. Filippi

e-mail: andreas.filippi@unibas.ch

M. L. Laine

Department of Oral Health Sciences, Section Periodontology, Academic Centre for Dentistry Amsterdam (ACTA), University of Amsterdam and VU University Amsterdam, Amsterdam,

The Netherlands

e-mail: m.laine@acta.nl gotten the attention and awareness of patients and dentists. Nevertheless, it is still a taboo subject. Since the oral cavity is the source of halitosis in most cases, a dental practitioner should be the first person to contact [1]. Before visiting a professional halitosis clinic, the majority of patients try to treat halitosis themselves [2•]. The most commonly used antihalitosis products are chewing gum, candies, and mouth rinse. However, it is known that these products only have a masking effect, and thus they are not able to influence the cause of bad breath [3]. More than half of the patients have, prior to their appointment at a halitosis clinic, already visited one or more general practitioners or medical specialists. Often, patients have a long history of suffering from halitosis, and the resulting social and psychological stress can be very high [2•].

The present review summarizes the diagnostics, prevalence, and current treatments of oral halitosis.

\section{Terminology}

Halitosis (Latin halitus: breath, vapor) describes an offensive, unpleasant smell of the breath, independent of the cause. In the literature, the terms halitosis, foetor ex ore [4], bad breath [5], breath odor [4], offensive breath [6], and oral malodor [7] are used synonymously. The recommendation of the international consensus group is to use the term 'halitosis' and to distinguish between intra-oral halitosis and extra-oral halitosis. This includes all of the cases of real halitosis [8••]. Real halitosis means that a distinctive bad breath can be recognized, which exceeds the socially accepted level. In the case of intraoral halitosis, the source lies within the mouth, and in case of extra-oral halitosis outside the mouth. Furthermore, extra-oral halitosis can be subdivided into blood-borne and non-bloodborne halitosis $[8 \bullet \bullet]$. In addition to real halitosis, psychogenic halitosis may be diagnosed [9]. Typically, the patient perceives a smell that is neither objectively verifiable nor measurable. After professional assessment and diagnosis, in patients with pseudo-halitosis the situation improves in contrast 
to patients with halitophobia. Even after intensive information and education, halitophopic patients persist in believing that they have bad breath. Halitosis caused by dietary factors such as garlic and onions is called temporary or transient halitosis $[8 \bullet \bullet]$. This classification is a slight simplification based on the International Classification of Halitosis published by Yaegaki and Coil [9], Coil et al. [10], and Miyazaki et al. [11] (Table 1).

\section{Causes}

Halitosis can have multifactorial causes. Nevertheless, in 80 $90 \%$ of halitosis the source can be found in the oral cavity [1]. Anaerobic, mainly Gram-negative, bacteria degrade organic substances (e.g., saliva, food debris, desquamated epithelial cells) into primarily volatile sulphur compounds (VSCs) [13]. These include hydrogen sulfide $\left(\mathrm{H}_{2} \mathrm{~S}\right)$, methyl mercaptan $\left(\mathrm{CH}_{3} \mathrm{SH}\right)$, dimethyl sulfide $\left(\left[\mathrm{CH}_{3}\right]_{2} \mathrm{~S}\right)$ and other minor component sulfides [12]. The VSCs producing bacteria are commonly found at the bottom of the micro-furrows and crypts on the dorso-posterior surface of the tongue. These bacteria have also been associated with gingivitis and periodontitis $[13,14]$. Further possible causes of intra-oral halitosis are gingivitis, periodontitis, open caries lesions, inadequate dental restorations, poor oral and denture hygiene, as well as local infections such as pericoronitis, periimplantitis, or candidiasis $[1,15]$. Additionally, there are co-factors that significantly influence halitosis, including reduced salivary flow rate, stress, smoking, mouth breathing, unbalanced diet, low daily amount of water, and coffee or alcohol consumption [16-18].

Extra-oral halitosis is uncommon [2•,19] and is mostly found in the ear, nose, and throat area or, in rare cases, in the gastrointestinal tract [15, 20, 21].

As the oral cavity is the main source of halitosis, general dental practitioners should be able to manage patients with intra-oral halitosis.

\section{Diagnosis}

A complete medical, dental, and halitosis history are indispensable. The medical history focuses on current medications and systemic diseases. The presence of nasal obstruction, mouth breathing, report of snoring and sleep apnea, postnasal drip, allergy, tonsillitis, tonsilloliths, dysphagia, previous ear, nose and throat encounters, types of food typically eaten, as well as vitamin A, B, C, D, and zinc-containing food intake are recorded. The dental history includes questions assessing the frequency of dental visits, the presence and maintenance of dental prosthesis as well as the frequency and the instruments used for tooth brushing, interdental cleaning, or tongue brushing/scraping and other dental products being used. Furthermore, a specific halitosis history should be taken $[8 \cdot \bullet$. A specifically designed halitosis questionnaire is given to the patient to complete before the first appointment. The questionnaire of the Halitosis Clinic of the University of Basel, Switzerland, can be found online (http://www. andreas-filippi.ch). This general and detailed halitosis questionnaire gives information about type, frequency, time of day and extent of halitosis, therapies already carried out through physicians, dentists, oral hygienists or self-treatment, resulting psychological stress, as well as typical halitosis cofactors such as dietary and smoking habits [22]. The question "How do you know that you have halitosis?" (options: selfreported, others have reported by straight or indirect way) gives an indication of possible existing psychogenic halitosis $[8 \cdot \bullet, 9]$. The questionnaire also serves as a starting point for the first appointment with the patient [2•].

\section{Clinical Examination}

The oral examination focuses on the predilection sites of intraoral halitosis. Periodontal screening is performed and the need

Table 1 Classification of different types of halitosis

\begin{tabular}{ll}
\hline Type of halitosis & Definition \\
\hline $\begin{array}{l}\text { Real halitosis } \\
\text { Temporary halitosis }\end{array}$ & $\begin{array}{l}\text { Obvious malodor with intensity beyond socially acceptable level and/or affecting personal relationships } \\
\text { Malodor caused by food and dietary factors such as garlic or morning bad breath } \\
\text { Intra-oral halitosis }\end{array}$ \\
& $\begin{array}{l}\text { The source lies within the mouth } \\
\text { malfunction of oral tissues (e.g., periodontal disease) }\end{array}$ \\
& $\begin{array}{l}\text { The condition is influenced by co-factors (e.g., medication, smoking, stress) } \\
\text { The source lies outside the mouth } \\
\text { Extra-oral halitosis: blood-borne }\end{array}$ \\
$\begin{array}{l}\text { Extra-oral halitosis: non-blood-borne } \\
\text { Psychogenic halitosis }\end{array}$ & $\begin{array}{l}\text { The malodor originates from nasal, paranasal, or laryngeal regions, or the pulmonary or upper digestive tract } \\
\text { Obvious malodor is not perceived by others but the patient complains of its existence. No physical or social } \\
\text { evidence exists for the presence of halitosis }\end{array}$ \\
Pseudo-halitosis & Condition is improved by counselling and simple oral hygiene measures \\
Halitophobia & The patient persists in believing they suffer from halitosis even after treatment of halitosis or pseudo-halitosis \\
\hline
\end{tabular}


for periodontal treatment and oral hygiene are assessed. Further examination of the oral and pharyngeal soft tissues (particularly coating of the tongue, Waldeyer's ring, and excretory ducts of the salivary glands) as well as dental fillings and restorations is performed $[2 \bullet]$.

\section{Measurements}

For the clinical detection of halitosis, the international consensus group recommends two primary methods [ $\left.8 \bullet^{\bullet}\right]$ : organoleptic measurement and instrumental measurements.

\section{Organoleptic Measurement}

Organoleptic measurement implies a subjective sensory test scored on the basis of the examiner's perception of a patient's breath odor $[8 \cdot \bullet, 9]$. The organoleptic assessment is easy and cheap and reflects an everyday situation [23]. One of the requirements is that the examiner has a good sense of smell [24•]. This can easily be verified by using the Smell Identification Test ${ }^{\mathrm{TM}}$ (Sensonics Inc., Haddon Heights, NJ, USA), which can reveal a person's ability to distinguish different odors $[8 \bullet \bullet, 24 \bullet]$. Further, the capacity to detect odors at low concentrations can be tested by sniffing series of dilutions of substances [24•]. It is recommended to have a second judge available, especially when dealing with patients suffering from psychogenic halitosis, as a second opinion is helpful $[8 \bullet \cdot]$. Several scoring methods have been described. The simplest one is a yes or no decision performed at different distances from the patient's mouth $(10,30$, and $100 \mathrm{~cm})[25$, 26]. For more experienced examiners, the widely used 6-point scale is recommended. It describes the severity of the perceived odor at a defined distance $[9,27,28]$.

The most important disadvantage of organoleptic assessment is the poor inter- and intra-examiner correlation of reliability and reproducibility [29•]. Furthermore, because of the subjectivity of this method [30,31], it is not always accepted by the patients, especially by those suffering from psychogenic halitosis [29•]. There are also some criteria for the examiner. A person who smokes, is pregnant, has chronic allergies, or asthma is not suitable as an odor judge [24•].

\section{Instrumental Measurements}

This is an objective way to measure VSCs, which are the principal components of oral malodor. An instrumental detection is not mandatory but it can help to build a second opinion, to calibrate odor judges, or to build trust with the patient, especially with patients suffering from psychogenic halitosis [8••, 32]. There are two devices that can be recommended for use at a dental practice: the Halimeter ${ }^{\circledR}$ and the OralChroma ${ }^{\mathrm{TM}}$ [29•].

The Halimeter ${ }^{\circledR}$ (Interscan, Chatsworth, CA, USA) is a portable sulfide monitor [31] that measures the total sulfide concentration. It is easy to use, the results are shown immediately, and the data are more reproducible than that of the organoleptic assessments. The disadvantages include the higher costs and that ethanol and other compounds can disturb the measurements [30, 33-35]. Furthermore, the sensitivity for $\mathrm{CH}_{3} \mathrm{SH}$ is five times lower than for $\mathrm{H}_{2} \mathrm{~S}$ and it is almost insensitive to $\left[\mathrm{CH}_{3}\right]_{2} \mathrm{~S}[9,36]$. Therefore, it is not suitable for measuring extra-oral blood-borne halitosis with $\left[\mathrm{CH}_{3}\right]_{2}$ origin [37].

The OralChroma ${ }^{\mathrm{TM}}$ (CHM-1, Abimedical, Kawasaki, Japan) is a chairside instrument bases on a gas chromatograph, which detects and discriminates the three most important VSCs [29•]. This information can be included in the diagnosis. For example, a high concentration of $\mathrm{CH}_{3} \mathrm{SH}$ compared to $\mathrm{H}_{2} \mathrm{~S}$ may indicate periodontitis [38], and an increased $\mathrm{H}_{2} \mathrm{~S}$ level may indicate a problem with oral hygiene. Further, increased levels of $\left[\mathrm{CH}_{3}\right]_{2}$ may indicate extra-oral halitosis [37]. The measurements are more reproducible and reliable than organoleptic measurements, and even extremely low gas concentrations can be detected. But the device is expensive and the results are not shown in real time; it takes $8 \mathrm{~min}$ before the results are shown. Recently, a new model has been introduced (CHM-2) with a reduced analysis time (4 min). To date, no literature is yet available about it [29•].

A disadvantage of both the Halimeter ${ }^{\circledR}$ and the OralChroma $^{\mathrm{TM}}$ is that they can only detect sulphur gases and not any other volatile components. Because other odorants (indoles, amines, acides), as well as VSCs, can contribute to halitosis, it is possible that an organoleptic rating is not always accompanied by a corresponding measurement on the Halimeter $^{\circledR}$ or OralChroma ${ }^{\mathrm{TM}}$ [29•]. In Table 2, the advantages and disadvantages of the above-mentioned measurements are summarized.

\section{Discussion}

For the organoleptic and instrumental detection of halitosis, there are many sampling methods described. Sniffing of the patient's breath air using the nose of the examiner (organoleptic measurement) is the usual technique for halitosis examination in daily practice. However, a sampling method, using a negative pressure syringe or a sample bag, was recommended by the international consensus group [8・•]. In comparison to directly sniffing the exhaled air of the patient during organoleptic assessment, sampling methods have the advantage of creating a higher degree of privacy for the patient and of receiving a more concentrated sample $[8 \cdot \bullet]$.

To receive the most reliable measurement results, the patient should receive instructions prior the first appointment $[8 \bullet \bullet, 9,11]$. At least $24 \mathrm{~h}$ before the appointment, the patient must not have eaten, for example, any onion or garlic or have cleaned her/his tongue. Furthermore, on the day of the investigation, the patient should refrain from using or doing 
Table 2 Advantages and disadvantages of organoleptic and instrumental measurements of halitosis

\begin{tabular}{lll}
\hline Measurement & Advantages & Disadvantages \\
\hline Organoleptic & No material costs & Subjective \\
& Chairside & Low reliability and reproducibility \\
& Easy to handle & \\
Halimeter ${ }^{(B)}$ & Chairside & Additional costs \\
& Easy to handle & Other compounds can disturb the measurement \\
& Results immediately displayed & Low detection of methyl mercaptan \\
& Objective & Detects only total sulphide concentration \\
OralChroma $^{\mathrm{TM}}(\mathrm{CHM}-1 / \mathrm{CHM}-2)$ & Relatively good reliability and reproducibility & \\
& Chairside & Relatively high costs \\
& Differentiation between three different gases & Single measurement takes 8 min/4 min \\
& Objective & Technically sensitive \\
& Relatively good reliability and reproducibility & Software not always reliable \\
\hline
\end{tabular}

anything that could cover the halitosis (e.g., perfumed cosmetic products, candies, chewing gums, mouth rinse) and should not smoke. In addition, ideally $4 \mathrm{~h}$ prior, the patient should not practice any oral hygiene, eat, or drink coffee $[2 \bullet$, $8 \bullet \bullet, 9,24 \bullet]$. The examiner should also follow the same restrictions for consumption of beverages and the use of cosmetics [2•, 22, 24•]. For the organoleptic evaluation of halitosis, it is advisable that the examiner calibrates her/his nose [24•, 39].

\section{Prevalence}

The prevalence of halitosis has been studied in different populations all over the world with a variety of techniques and cut-offs, but the prevalence of halitosis is still not wellestablished. Most of the prevalence studies are based on selfperceived breath odor and do not correlate well with other halitosis measurements [40]. Therefore, the results of selfperceived halitosis should be interpreted with caution. A total of $31 \%$ of American seniors have been reported to suffer from chronic or recurrent halitosis $(N=270)$ [41], $32 \%$ of Swiss adults reported experiencing halitosis sometimes or often $(N=$ 419) [25], and $45 \%$ of Indian dental students $(N=277)$ reported halitosis, with $>80 \%$ of them experiencing morning bad breath [42].

One study has investigated the prevalence of halitosis in the general Dutch population $(N=1,002,>16$ years old $)$, and reported that almost $90 \%$ of the population was regularly faced with a person having halitosis, $40 \%$ at least once a week [43].

Using organoleptic measurements, trained examiners recorded halitosis (score $\geq 2$ on a scale from 0 to $5[9,27,28]$ ) in $27.5 \%$ of a Chinese population $(N=2,000$, aged $15-$ 64 years) [44] and in $31.5 \%$ of a Swiss population $(N=$
419, aged 18-94 years) [25], whereas $85 \%$ of Swiss army recruits $(N=626)$ [45] were diagnosed with halitosis as classified by Seemann [46].

Instrumental measurements of halitosis may give an objective and exact value for halitosis, but it is important to notice that different studies have used various threshold levels for halitosis. On the basis of Halimeter ${ }^{\circledR}$, halitosis was established in 2,672 Japanese Government employees (aged 18-64 years), and $6-23 \%$ of the subjects showed VSC values above the suggested socially acceptable level of $75 \mathrm{ppb}$ at some time during the day [40]. With the same VSC cut-off level, a total of $23 \%$ of a Chinese population [44], $43 \%$ of Swiss army recruits [45], and $28 \%$ of a Swiss general population were diagnosed with halitosis [25]. However, with the thresholds of $\geq 110$ and $\geq 150 \mathrm{ppb}$, respectively, 4.3 and $1.2 \%$ of the Swiss and 35 and $20 \%$ of the Chinese population had halitosis [25, 44].

On the basis of OralChroma ${ }^{\circledR}$, measuring three different VSCs, $>60 \%$ of a Thai elderly population $(N=428$, mean age 68 years) have been diagnosed with halitosis [47].

In general, halitosis has been reported to be similarly prevalent in females and males [25, 40, 44]. However, some studies have found a higher prevalence of halitosis in women [44, 48]. Liu et al. found a higher prevalence in women only in the age group of 35-44 years old [44]. There are contradictory reports on the influence of age on halitosis; some studies have associated halitosis with increasing age [40, 48], whereas others have not [25, 44]. It is difficult to determine the actual influence of factors such as age and gender on halitosis, since breath odor may be influenced by many factors such as periodontal and dental status, dental hygiene, tongue coating, smoking, nutrition, level of education, and medication.

Prevalence of pseudohalitosis in the general population is not known but it has been reported to be about $16 \%$ in the patients visiting professional halitosis clinics $[1,2 \bullet]$. 


\section{Treatment}

The choice of treatment is made on the basis of the diagnosis and includes cause-related therapy [21]. The recommendations for the treatment of halitosis are based on publications by Yaegaki and Coil [9], Coil et al. [10], and Miyazaki et al. [11].

After detailed information and explanation of halitosis, each patient is given instructions for oral hygiene. Because the tongue coating is the most common cause of halitosis, the instructions also include mechanical tongue cleaning as a part of daily oral hygiene $[9,22,49]$. Various studies have shown that tongue cleaning leads to reduced levels of VSCs and thus to reduction of halitosis [50-53]. Many different tongue cleaners are available on the market. A tongue scraper can only remove the upper surface layer of the biofilm, which is why the effect of a tongue scraper is shorter in duration than the effect of a tongue brush [54]. Moreover, cleaning too hard with a tongue scraper, is a risk for tongue injury. Additionally, the effect of tongue cleaning may be extended using a tongue paste with active substances $[22,55]$. Any kind of electrical device for professional tongue cleaning is not recommended $[8 \cdot \bullet$. Animal experiments have shown that mechanical injuries of the tongue may be carcinogenic [56-58]. Therefore, detailed and comprehensive tongue cleaning instructions are necessary. Tongue cleaning should be carried out gently with low force at the posterior part of the tongue dorsum. Further, the lateral borders should not be cleaned because of the risk of traumatic injury [9].

If tongue cleaning alone is not sufficient, additional mouth rinse can be use $[2 \bullet, 8 \bullet \cdot$. As well as flavoring agents (e.g., mint) for masking odor, mouth rinses often contain antibacterial (e.g., chlorhexidine [59]) or neutralizing components (e.g., zinc [60]), which are able to absorb the VSCs or their precursors $[22,61]$, and are responsible for the therapeutic effect [62]. In commercial products, antibacterial and odorneutralizing agents are often combined. This leads to a strengthening or even synergistic effect $[22,61]$. Some patients may experience side effects such as discoloring and altered taste when chlorhexidine-containing mouth rinses are used for a long period $[9,60,63]$.

In the literature, there is some evidence that probiotics (Greek Pro bios: 'for life') not only have a positive effect in the gastrointestinal tract, but also in the oral cavity [64, 65]. With the help of chewing gums, bacteria such as Streptococcus salivarius and Lactobacillus salivarius are introduced into the oral cavity and are aimed at displacing halitosis-associated bacteria [66-69]. Despite promising results, further research is needed before probiotics can be used to treat oral halitosis $[70,71]$.

If halitosis originates from another oral cause such as gingivitis, periodontitis, caries, or insufficient dental restorations, corresponding therapy is initiated $[2 \bullet, 9]$. In addition, possible co-factors are addressed and, if necessary, adjusted. Sometimes, consultation with a physician is indicated [22]. If extra-oral halitosis is diagnosed, the patient is referred to appropriate specialists such as an otorhinolaryngologist or internist $[8 \bullet \bullet, 22]$.

If no halitosis is diagnosed upon the first examination, it is recommended that a second appointment at a different time of day should be arranged to avoid any effects of circadian rhythms. In the case of psychogenic halitosis, the diagnosis is communicated at the second appointment [72]. Dealing with patients suffering from halitophobia is difficult and requires a lot of experience [10]. Not every patient takes the advice given in psychological counseling immediately. Any dentist who offers a halitosis consultation should work together with a psychologist or psychiatrist and, if the patient agrees, refer her/him [22, 72].

\section{Conclusion}

Halitosis is a common problem affecting approximately $30 \%$ of the general population. In many cases, halitosis originates from a combination of oral causes and existing co-factors. Therefore, a comprehensive diagnosis is required. In addition to a halitosis history, an extensive evaluation of the patients' general health is also taken. A full investigation of the predilection sites of intra-oral halitosis as well as the pharyngeal soft tissue is performed. This is necessary to exclude an extraoral cause. Furthermore, an organoleptic and instrumental measurement of the patient's breath air is carried out.

If intra-oral halitosis is diagnosed, a cause-related treatment is conducted. In most cases, tongue cleaning is the treatment of choice. Additional mouth rinses with proven efficacy (e.g., with a chlorhexidine and zinc formulation) can be used.

The treatment of patients with halitosis should be performed according to a standardized scheme. Patients with extra-oral halitosis and halitophobia must be referred to appropriate specialists such as an otorhinolaryngologist, internist, psychologist, or psychiatrist.

With the consistent implementation of the abovementioned diagnostic and therapeutic concepts, a high level of treatment success can be achieved $[2 \bullet]$.

\section{Compliance with Ethics Guidelines}

Conflict of Interest Andrea Zürcher and Andreas Filippi declare that they have no conflict of interest.

Marja L. Laine is supported in part by a grant from the University of Amsterdam, Amsterdam, The Netherlands, for the focal point "Oral infection and inflammation."

Human and Animal Rights and Informed Consent No human or animal studies performed by the authors:

This article does not contain any studies with human or animal subjects performed by any of the authors. 


\section{References}

Papers of particular interest, published recently, have been highlighted as:

- Of importance

- Of major importance

1. Quirynen M, Dadamio J, Van den Velde S, De Smit M, Dekeyser C, Van Tornout M, et al. Characteristics of 2000 patients who visited a halitosis clinic. J Clin Periodontol. 2009;36:970-5.

2. Zürcher A, Filippi A. Findings, diagnoses and results of a halitosis clinic over a seven year period. Schweiz Monatsschr Zahnmed. 2012;122:205-16. This is a retrospective study which analyzed data from 465 patients of the Halitosis Clinic at the University of Basel.

3. Quirynen M, Zhao H, van Steenberghe D. Review of the treatment strategies for oral malodour. Clin Oral Investig. 2002;6:1-10.

4. Scully C, El-Maaytah M, Porter SR, Greenman J. Breath odor: etiopathogenesis, assessment and management. Eur J Oral Sci. 1997;105:287-93.

5. Goldberg S, Kozlovsky A, Gordon D, Gelernter I, Sintov A, Rosenberg M. Cadaverine as a putative component of oral malodor. J Dent Res. 1994;73:1168-72.

6. McDowell JD, Kassebaum DK. Diagnosing and treating halitosis. J Am Dent Assoc. 1993;124:55-64.

7. Tessier JF, Kulkarni GV. Bad breath: etiology, diagnosis and treatment. Oral Health. 1991;81:19-22. 24.

8.• Seemann R, Conceicao MD, Filippi A, Greenman J, Lenton P, Nachnani S, et al. Halitosis management by the general dental practitioner-results of an international consensus workshop. J Breath Res. 2014;8:017101. This article summarizes the results of a consensus workshop of international authorities with the aim of reaching a consensus on general guidelines on how to assess and diagnose patients' breath odor and on the treatment of halitosis.

9. Yaegaki K, Coil JM. Examination, classification, and treatment of halitosis; clinical perspectives. J Can Dent Assoc. 2000;66:257-61.

10. Coil JM, Yaegaki K, Matsuo T, Miyazaki H. Treatment needs (TN) and practical remedies for halitosis. Int Dent J. 2002;52:187-91.

11. Miyazaki H, Arao M, Okamura K, Kawaguchi Y, Toyofuku A, Hoshi K, et al. Tentative classification of halitosis and its treatment needs. Niigata Dent J. 1999;32:7-11.

12. Krespi YP, Shrime MG, Kacker A. The relationship between oral malodor and volatile sulfur compound-producing bacteria. Otolaryngol Head Neck Surg. 2006;135:671-6.

13. Apatzidou AD, Bakirtzoglou E, Vouros I, Karagiannis V, Papa A, Konstantinidis A. Association between oral malodour and periodontal disease-related parameters in the general population. Acta Odontol Scand. 2013;71:189-95.

14. Sterer N, Rosenberg M. Breath odors. Origin, diagnosis and management. Berlin: Springer; 2011. p. 5-75.

15. Delanghe G, Bollen C, Desloovere C. Halitosis-foetor ex ore. Laryngorhinootologie. 1999;78:521-4.

16. Rosenberg M, Knaan T, Cohen D. Association among bad breath, body mass index, and alcohol intake. J Dent Res. 2007;86:997-1000

17. Christen AG. The impact of tobacco use and cessation on oral and dental diseases and conditions. Am J Med. 1992;93:25-31.

18. Van den Broek AM, Feenstra L, de Baat C. A review of the current literature on aetiology and measurement methods of halitosis. J Dent. 2007;35:627-35.

19. Tonzetich J. Production and origin of oral malodor: a review of mechanisms and methods of analysis. J Periodontol. 1977;48:13-20.

20. Lambrecht JT. Extra-oral causes. In: Filippi A, editor. Halitosis. Berlin: Quintessenz; 2011. p. 67-74.
21. Kislig K, Wilder-Smith CH, Bornstein MM, Lussi A, Seemann R. Halitosis and tongue coating in patients with erosive gastroesophageal reflux disease versus nonerosive gastroesophageal reflux disease. Clin Oral Investig. 2013;17:159-65.

22. Filippi A. Halitosis. Berlin: Quintessenz; 2006.

23. Shimura M, Yasuno Y, Iwakura M, Shimada Y, Sakai S, Suzuki K, et al. A new monitor with a zinc-oxide thin film semiconductor sensor for the measurement of volatile sulfur compounds in mouth air. J Periodontol. 1996;67:396-402.

24. Greenman J, Lenton P, Seemann R, Nachnani S. Organoleptic assessment of halitosis for dental professionals-general recommendations. J Breath Res. 2014;8:017102. This article describes the organoleptic assessment of halitosis and its importance for dental professionals.

25. Bornstein MM, Kislig K, Hoti BB, Seemann R, Lussi A. Prevalence of halitosis in the population of the city of Bern, Switzerland: a study comparing self-reported and clinical data. Eur J Oral Sci. 2009;117:261-7.

26. Seemann R. Halitosis - a solvable problem [in German]. Zahnärztlicher Anz München. 2001;47:4-7.

27. Rosenberg M. Clinical assessment of bad breath: current concepts. J Am Dent Assoc. 1996;127:475-82.

28. Murata T, Yamaga T, lida T, Miyazaki H, Yaegaki K. Classification and examination of halitosis. Int Dent J. 2002;52:181-6.

29. Laleman I, Dadamio J, De Geest S, Dekeyser C, Quirynen M. Instrumental assessment of halitosis for the general dental practitioner. J Breath Res. 2014;8:017103. This paper summarizes and compares the most discussed tools used for the assessment of halitosis. It shows their advantages and disadvantages and provides guidance for use by general dental practitioners.

30. Rosenberg M, Kulkarni GV, Bosy A, McCulloch CA. Reproducibility and sensitivity of oral malodor measurements with a portable sulphide monitor. J Dent Res. 1991;70:1436-40.

31. Rosenberg M, Septon I, Eli I, Bar-Ness R, Gelernter I, Brenner S, et al. Halitosis measurement by an industrial sulphide monitor. J Periodontol. 1991;62:487-9.

32. Vandekerckhove B, Van den Velde S, De Smit M, Dadamio J, Teughels W, Van Tornout M, et al. Clinical reliability of nonorganoleptic oral malodour measurements. J Clin Periodontol. 2009;36:964-9.

33. Baharvand M, Maleki Z, Mohammadi S, Alavi K, Moghaddam EJ. Assessment of oral malodor: a comparison of the organoleptic method with sulfide monitoring. J Contemp Dent Pract. 2008;9: 76-83.

34. Murata T, Rahardjo A, Fujiyama Y, Yamaga T, Hanada M, Yaegaki $\mathrm{K}$, et al. Development of a compact and simple gas chromatography for oral malodor measurement. J Periodontol. 2006;77:1142-7.

35. Van Steenberghe D, Avontroodt P, Peeters W, Pauwels M, Coucke W, Lijnen A, et al. Effect of different mouthrinses on morning breath. J Periodontol. 2001;72:1183-91.

36. Furne J, Majerus G, Lenton P, Springfield J, Levitt DG, Levitt MD. Comparison of volatile sulfur compound concentrations measured with a sulfide detector vs. gas chromatography. J Dent Res. 2002;81:140-3.

37. Tangerman A, Winkel EG. Intra- and extra-oral halitosis: finding of a new form of extra-oral blood-borne halitosis caused by dimethyl sulphide. J Clin Periodontol. 2007;34:748-55.

38. Yaegaki K, Sanada K. Volatile sulfur compounds in mouth air from clinically healthy subjects and patients with periodontal disease. J Periodontal Res. 1992;27:233-8.

39. Evirgen S, Kamburoğlu K, Gulsahi A. Effect of clinician's experience, age, gender and calibration on the assessment of halitosis. Oral Health Prev Dent. 2013;11:17-22.

40. Miyazaki H, Sakao S, Katoh Y, Takehara T. Correlation between volatile sulphur compounds and certain oral health measurements in the general population. J Periodontol. 1995;66:679-84. 
41. Loesche WJ, Grossman N, Dominguez L, Schork MA. Oral malodour in the elderly. In: van Steenberghe D, Rosenberg M, editors. Bad breath: a multidisciplinary approach. Leuven: Leuven University Press; 1996. p. 181-94.

42. Setia S, Pannu P, Gambhir RS, Galhotra V, Ahluwalia P, Sofat A. Correlation of oral hygiene practices, smoking and oral health conditions with self perceived halitosis amongst undergraduate dental students. J Nat Sci Biol Med. 2014;5:67-72.

43. De Jongh A, van Wijk AJ, Horstman M, de Baat C. Attitudes towards individuals with halitosis: an online cross sectional survey of the Dutch general population. Br Dent J. 2014;216:E8.

44. Liu XN, Shinada K, Chen XC, Zhang BX, Yaegaki K, Kawaguchi Y. Oral malodor-related parameters in the Chinese general population. J Clin Periodontol. 2006;33:31-6.

45. Bornstein MM, Stocker BL, Seemann R, Bürgin WB, Lussi A. Prevalence of halitosis in young male adults: a study in Swiss army recruits comparing self-reported and clinical data. J Periodontol. 2009;80:24-31.

46. Seemann R. Measurement of halitosis. In: Filippi A, editor. Halitosis. Patients with oral malodor in daily dental practice [in German]. Berlin: Quintessence; 2006. p. 39-50.

47. Samnieng P, Ueno M, Shinada K, Zaitsu T, Kawaguchi Y. Daily variation of oral malodour and related factors in communitydwelling elderly Thai. Gerodontology. 2012;29:e964-71.

48. Nadanovsky P, Carvalho LB, Ponce de Leon A. Oral malodour and its association with age and sex in a general population in Brazil. Oral Dis. 2007;13:105-9.

49. Kuo YW, Yen M, Fetzer S, Lee JD. Toothbrushing versus toothbrushing plus tongue cleaning in reducing halitosis and tongue coating: a systematic review and meta-analysis. Nurs Res. 2013;62:422-9.

50. O'Leary TJ, Drake RB, Naylor JE. The plaque control record. J Periodontol. 1972;43:38.

51. Outhouse TL, Al-Alawi R, Fedorowicz Z, Keenan JV. Tongue scraping for treating halitosis. Cochrane Database Syst Rev. 2006;2, CD005519.

52. Lee SS, Zhang W, Li Y. Halitosis update: a review of causes, diagnoses, and treatments. J Calif Dent Assoc. 2007;35:258-68.

53. Van der Sleen MI, Slot DE, Van Trijffel E, Winkel EG, Van der Weijden GA. Effectiveness of mechanical tongue cleaning on breath odour and tongue coating: a systematic review. Int J Dent Hyg. 2010;8:258-68.

54. Hoshi K, Van Steenberghe D. The effect of tongue brushing or toothpaste application on oral malodour reduction. Bad breath. A multidisciplinary approach. Leuven: University Press; 1996. p. 255-64.

55. Dadamio J, Laleman I, Quirynen M. The role of toothpastes in oral malodor management. Monogr Oral Sci. 2013;23:45-60.
56. Kameyama T. Experimentally induced tongue cancer by application of 4-Nitroyuinoline 1-oxide. J Jpn Stomatol Coc. 1969;18: 609-24.

57. Odajima T, Fujita K, Kaku T, Okuyama T. Effect of frequent application of carcinogen upon lingual carcinogenesis experiment. J Jpn Stomatol Soc. 1979;25:523-6.

58. Fujita K, Kaku T, Sasaki M, Onoé T. Experimental production of lingual carcinomas in hamsters: tumor characteristics and site of formation. J Dent Res. 1973;52:1176-85.

59. Rosenberg M, Gelernter I, Barki M, Bar-Ness R. Day-long reduction of oral malodor by a two-phase oil:water mouthrinse as compared to chlorhexidine and placebo rinses. J Periodontol. 1992;63: 39-43.

60. Yaegaki K, Suetaka T. Periodontal disease and precursors of oral malodorous components. J Dent Health. 1989;39:733-41.

61. Blom T, Slot DE, Quirynen M, Van der Weijden GA. A review of the current literature on aetiology and measurement methods of halitosis. J Dent. 2007;35:627-35.

62. Dadamio J, Van Tournout M, Teughels W, Dekeyser C, Coucke W, Quirynen M. Efficacy of different mouthrinse formulations in reducing oral malodour: a randomized clinical trial. J Clin Periodontol. 2013;40:505-13.

63. Jones CG. Chlorhexidine: is it still the gold standard? Periodontol 2000. 1997;15:55-62.

64. Bonifait L, Chandad F, Grenier D. Probiotics for oral health: myth or reality? J Can Dent Assoc. 2009;75:585-90.

65. Twetman S, Stecksén-Blicks C. Probiotics and oral health effects in children. Int J Paediatr Dent. 2008;18:3-10.

66. Burton JP, Chilcott CN, Moore CJ, Speiser G, Tagg JR. A preliminary study of the effect of probiotic Streptococcus salivarius K12 on oral malodour parameters. J Appl Microbiol. 2006;100:754-64.

67. Horz HP, Meinelt A, Houben B, Conrads G. Distribution and persistence of probiotic Streptococcus salivarius K12 in the human oral cavity as determined by real-time quantitative polymerase chain reaction. Oral Microbiol Immunol. 2007;22:126-30.

68. Iwamoto T, Suzuki N, Tanabe K, Takeshita T, Hirofuji T. Effects of probiotic Lactobacillus salivarius WB21 on halitosis and oral health: an open-label pilot trial. Oral Surg Oral Med Oral Pathol Oral Radiol Endod. 2010;110:201-8.

69. Wescombe PA, Hale JD, Heng NC, Tagg JR. Developing oral probiotics from Streptococcus salivarius. Future Microbiol. 2012;7:1355-71.

70. Pradeep K, Kuttappa MA, Prasana KR. Probiotics and oral health: an update. SADJ. 2014;69:20-4.

71. Rao Y, Lingamneni B, Reddy D. Probiotics in oral health-a review. J N J Dent Assoc. 2012;83:28-32.

72. Nagel D, Lutz C, Filippi A. Halitophobia-an under-recognized clinical picture. Schweiz Monatsschr Zahnmed. 2006;116:57-64. 